\title{
Clinical Value of C-Reactive Protein/Platelet Ratio in Neonatal Sepsis: A Cross-Sectional Study
}

\author{
Xiaojuan $\mathrm{Li}^{1}$ \\ Tiewei $\mathrm{Li}^{1}$ \\ jingjing Wang ${ }^{2}$ \\ Yichuan Feng' \\ Chong Ren' \\ Zhe Xu' \\ Junmei Yang' \\ Qian Zhang ${ }^{3}$ \\ Caiyan $\mathrm{An}^{4}$
}

'Zhengzhou Key Laboratory of Children's Infection and Immunity, Children's Hospital Affiliated to Zhengzhou University, Henan Children's Hospital, Zhengzhou Children's Hospital,

Zhengzhou, 4500 I8, People's Republic of China; ${ }^{2}$ Department of Neonatology, Ordos Central Hospital, Ordos, 01700 , People's Republic of China; ${ }^{3}$ The Center for New Drug Safety Evaluation and Research, Inner Mongolia Medical University, Hohhot, People's Republic of China; ${ }^{4}$ Clinical Research Center of the Affiliated Hospital, Inner Mongolia Medical University, Hohhot, People's Republic of China
Correspondence: Tiewei Li Zhengzhou Key Laboratory of Children's Infection and Immunity, Children's Hospital Affiliated to Zhengzhou University, Henan Children's Hospital,

Zhengzhou Children's Hospital, 33M

Waihuan East Road, Longhu, Zhengzhou,

4500 I8, People's Republic of China

Tel +86371855I5779

Email litieweind@63.com

Jingjing Wang

Ordos Central Hospital, No. 23, Yijinholo

Street, Dongsheng District, Ordos,

0I7000, People's Republic of China

Tel +86-I5924482258

Email onlyonecoco@I63.com
Purpose: C-reactive protein (CRP) level and platelet (PLT) count have been demonstrated to be independent risk factor for neonatal sepsis. However, no data is currently available in regarding the association between CRP-to-PLT ratio (CPR) and neonatal sepsis.

Methods: A total of 1048 neonates with suspected sepsis were enrolled in this study. Complete clinical and laboratory data were collected. CPR was calculated as CRP (mg/L)/ PLT $\left(10^{7}\right.$ cells/L). Multivariate logistic regression analysis was performed to identify the potential independent risk factors of neonatal sepsis. Receiver operating characteristic (ROC) curve analysis was used to evaluate the prediction accuracy of CPR in predicting neonatal sepsis.

Results: Neonates with sepsis had a higher CPR. CPR also showed a gradual increase in the infection, mild sepsis and severe sepsis groups. Multivariate analysis revealed that CPR was a significant independent predictor of the presence of neonatal sepsis (odds ratio [OR], 1.015; 95\% confidence interval [CI], 1.008-1.022, $\mathrm{P}<0.001)$ and severe sepsis $(\mathrm{OR}, 1.002 ; 95 \%$ $\mathrm{CI}, 1.000-1.003, \mathrm{P}=0.007)$. ROC curve revealed showed that CPR had a welldiscriminatory power in predicting sepsis (area under curve [AUC], 0.68; 95\% CI, 0.65$0.72, \mathrm{P}<0.001$ ) and severe sepsis (AUC, 0.68; 95\% CI, 0.65-0.72, P < 0.001).

Conclusion: The present study demonstrated that a higher CPR is an independent predictor of the presence and severity of neonatal sepsis.

Keywords: C-reactive protein-to-platelet ratio, neonatal sepsis, risk factor, severe sepsis

\section{Instruction}

Neonatal sepsis remains a serious and life-threatening disease in infants worldwide, despite the improvements in neonatology. Previously published data revealed that neonatal sepsis constituted $15.2 \%$ of deaths in the neonatal period worldwide, ${ }^{1}$ and is a major cause of infant mortality. ${ }^{2,3}$ The gold-standard for diagnosis of neonatal sepsis is blood culture. However, blood culture poses several challenges, such as a long laboratory turnaround time, an inadequate volume of blood and pre-hospital antimicrobial therapy. ${ }^{4,5}$ In addition, the clinical signs of neonatal sepsis are multiple and non-specific, including bradycardia, temperature instability, diminished spontaneous activity and respiratory distress. ${ }^{6}$ Therefore, identifying rapid, sensitive, and specific new biomarkers is critical.

C-reactive protein (CRP) is an acute-phase protein produced by the liver and closely associated with systemic inflammatory status. ${ }^{7}$ In 1988 , Povoa et $\mathrm{al}^{8}$ reported for the first time that CRP was an indicator of sepsis. Subsequently, multiple studies confirmed that CRP was an important predictor and risk factor for sepsis. ${ }^{9,10}$ CRP evaluation was also one of the most investigated and used 
laboratory tests for diagnosing neonatal sepsis, and higher CRP levels were associated with an increased risk of sepsis. ${ }^{11}$ However, CRP was not efficiently validated as a screening biomarker. ${ }^{12}$ Platelets (PLTs) are circulating blood cells, which play an important role in haemostasis and coagulation. PLTs can release inflammatory cytokines, interact with endothelial cells and contribute to the formation of a microthrombus, eventually leading to multiple organ failure. ${ }^{13}$ Studies have demonstrated that PLTs are involved in the pathogenesis of sepsis and contribute to its complications. $^{14,15}$ Clinical studies have reported that patients with severe sepsis have a lower PLT count, ${ }^{16}$ which is associated with enhanced mortality and a more disturbed host response. ${ }^{17}$ The CRP-to-PLT ratio (CPR), based on CRP levels and PLT counts, indicates not only the inflammation but also the coagulation status. However, no data is currently available regarding the association of CPR with the neonatal sepsis. Therefore, this study aimed to assess the relationship between CPR and neonatal sepsis.

\section{Materials and Methods Study Design and Patient Population}

From January 2016 to February 2020, a total 1098 consecutive neonates with suspected sepsis in Henan Children's Hospital (Zhengzhou, China) were enrolled in this study. The inclusion criteria were described as follows: (1) neonates with suspected sepsis and (2) neonates aged 1-28 days. Neonates with the following conditions were excluded from this study: (1) with haematological system diseases, malignancies or major congenital malformations and (2) incomplete clinical and laboratory data at admission. The study protocol complied with the Declaration of Helsinki and was approved by the ethics review board of the hospital. Informed consent was not required because the data were anonymised.

\section{Clinical Definition}

According to the recommendations of the International Pediatric Sepsis Consensus, ${ }^{18}$ neonatal sepsis is defined as suspected or proven infection accompanied by $\geq 2$ systemic inflammatory response syndrome (SIRS) criteria, one being an abnormal body temperature or leukocyte count. The criteria for SIRS are as follows: (1) body temperature $>$ $38.5^{\circ} \mathrm{C}$ or $<36^{\circ} \mathrm{C}$; (2) mean heart rate $>2 \mathrm{SD}$ above normal for age in the absence of external stimuli or unexplained persistent elevation in children aged $<1$ year old or mean heart rate $<10$ th percentile for age, or unexplained persistent depression over a $0.5 \mathrm{hr}$; (3) mean respiratory rate of $>2$ SD above normal for age or in the presence of mechanical ventilation; and (4) abnormal leukocyte count or $>10 \%$ immature neutrophils. Severe sepsis is defined as sepsis accompanied by one of the following: cardiovascular organ dysfunction or acute respiratory distress syndrome or $\geq 2$ organ dysfunctions. Detailed information can be obtained from the International Pediatric Sepsis Consensus. $^{18}$

\section{Data Collection and Biochemical Analyses}

All clinical and laboratory data were obtained from medical records, including age; gender; weight; temperature; respiratory rate; heart rate; systolic blood pressure; diastolic blood pressure and the levels of procalcitonin (PCT), CRP, white blood cells (WBCs), neutrophils, PLTs, aspartate aminotransferase (AST), alanine aminotransferase (ALT), blood urea nitrogen (BUN), creatinine (CREA) and uric acid (UA). The detection methods of these laboratory indices are described in our previous published study. ${ }^{19}$ CRP level $<0.8 \mathrm{mg} / \mathrm{L}$ or PCT level $>100 \mathrm{ng} /$ $\mathrm{mL}$ or $<0.02 \mathrm{ng} / \mathrm{mL}$ were considered as $0.7 \mathrm{mg} / \mathrm{L}, 101 \mathrm{ng} /$ $\mathrm{mL}$ and $0.01 \mathrm{ng} / \mathrm{mL}$, respectively. CPR was calculated as $\mathrm{CRP} / \mathrm{PLT} * 10^{3}$.

\section{Statistical Analysis}

Continuous data were expressed as the mean \pm standard deviation (SD) or medians (interquartile range) and analysed using independent Student's $t$-tests, one-way analysis of variance (ANOVA) or Mann-Whitney $U$-test, according to their distribution. Categorical variables were presented as percentages $(n, \%)$ and analysed using the chi-square or Fisher exact tests. The Pearson or Spearman correlation analysis was performed to determine the correlation between two continuous variables. Univariate and multivariate logistic regression analyses were performed to evaluate whether CPR was an independent risk factor for the presence and severity of neonatal sepsis. Variables with a $\mathrm{P}$ value $<0.05$ in the univariate logistic analysis were included in the multiple regression analysis. The prediction accuracy was evaluated using the area under the receiver operating characteristic (ROC) curves. All statistical analyses were performed using SPSS 22.0 (SPSS Inc., Chicago, Illinois, USA). A two-sided P value $<0.05$ was considered statistically significant. 
Table I Basic Characteristics of Study Subjects

\begin{tabular}{|c|c|c|c|c|}
\hline \multirow[t]{2}{*}{ Variables } & \multirow[t]{2}{*}{ Infection $(n=449)$} & \multirow[t]{2}{*}{ Sepsis $(n=599)$} & \multicolumn{2}{|c|}{ Sepsis } \\
\hline & & & Mild Sepsis $(n=256)$ & Severe Sepsis $(n=343)$ \\
\hline Age (days) & $7.0(4.0,12.0)$ & $11.0(6.0,17.0)^{\mathrm{a}}$ & $11.0(6.0,18.0)^{c}$ & $10.0(6.0,16.0)^{d}$ \\
\hline Male, n (\%) & $262(58.4 \%)$ & $375(62.6 \%)$ & $165(64.5 \%)$ & $210(61.2 \%)$ \\
\hline Weight (kg) & $3.3 \pm 0.5$ & $3.2 \pm 0.6^{\mathrm{a}}$ & $3.3 \pm 0.6$ & $3.1 \pm 0.7^{\text {bd }}$ \\
\hline Temperature $\left({ }^{\circ} \mathrm{C}\right)$ & $37.0 \pm 0.5$ & $37.3 \pm 0.8^{\mathrm{a}}$ & $37.4 \pm 0.7^{c}$ & $37.3 \pm 0.8^{d}$ \\
\hline Respiratory (rate/minute) & $46.5 \pm 7.6$ & $49.7 \pm 10.1^{a}$ & $49.4 \pm 9.6^{c}$ & $50.0 \pm 10.4^{d}$ \\
\hline Heart rate (bpm) & $142.7 \pm 16.1$ & $150.3 \pm 18.0^{\mathrm{a}}$ & $149.6 \pm 17.7^{c}$ & $150.8 \pm 18.3^{d}$ \\
\hline $\mathrm{SBP}(\mathrm{mm} \mathrm{Hg})$ & $76.4 \pm 7.1$ & $76.3 \pm 8.0$ & $79.3 \pm 5.6^{c}$ & $74.1 \pm 9.0^{\text {bd }}$ \\
\hline DBP $(\mathrm{mm} \mathrm{Hg})$ & $46.6 \pm 7.3$ & $46.2 \pm 7.8$ & $47.7 \pm 7.6$ & $45.0 \pm 7.7^{\mathrm{bd}}$ \\
\hline \multicolumn{5}{|l|}{ Laboratory tests } \\
\hline PCT (ng/mL) & $0.14(0.09,0.22)$ & $0.28(0.13,1.23)^{\mathrm{a}}$ & $0.22(0.11,0.66)^{c}$ & $0.36(0.65,1.88)^{\text {bd }}$ \\
\hline CRP (mg/L) & $0.7(0.7,0.7)$ & $0.7(0.7,12.8)^{\mathrm{a}}$ & $0.7(0.7,8.1)^{c}$ & $0.7(0.7,17.5)^{\mathrm{bd}}$ \\
\hline WBC $\left(10^{9}\right.$ cells $\left./ \mathrm{L}\right)$ & $10.0(8.1,12.2)$ & $10.0(7.3,13.9)$ & $9.4(7.4,12.6)$ & $10.7(7.0,15.8)^{\mathrm{bd}}$ \\
\hline Neutrophils ( $10^{9}$ cells/L) & $4.18(3.13,5.82)$ & $4.89(3.02,8.33)^{\mathrm{a}}$ & $4.47(3.01,6.95)$ & $5.52(3.05,9.37)^{\text {bd }}$ \\
\hline PLT ( $10^{9}$ cells $\left./ \mathrm{L}\right)$ & $293.0(229.5,365.0)$ & $266.0(170,364.0)^{\mathrm{a}}$ & $297.0(216.2,368.7)$ & $237.0(121.0,359.0)^{\mathrm{bd}}$ \\
\hline CPR (mg/10 cells) & $2.49(1.95,3.56)$ & $4.8 \mathrm{I}(2.28,57.05)^{\mathrm{c}}$ & $3.10(2.15,26.06)^{c}$ & $8.97(2.63,96.60)$ bd \\
\hline AST (U/L) & $37.5(30.0,49.9)$ & $37.8(27.9,52.7)$ & $36.2(27.9,47.9)$ & $38.9(27.8,58.5)^{\mathrm{b}}$ \\
\hline ALT (U/L) & $25.1(20.0,33.4)$ & $28.6(22.1,37.9)^{\mathrm{a}}$ & $28.7(22.7,36.2)^{c}$ & $28.5(22.0,39.6)^{d}$ \\
\hline BUN (mM) & $2.2(1.4,3.3)$ & $3.0(1.9,4.2)^{\mathrm{a}}$ & $2.8(1.8,3.9)^{c}$ & $3.2(1.9,4.7)^{\mathrm{bd}}$ \\
\hline CREA $(\mu \mathrm{M})$ & $50.0(41.3,57.6)$ & $45.2(35.9,60.3)^{\mathrm{a}}$ & $43.3(34.7,53.2)^{c}$ & $47.7(37.2,64.3)^{\mathrm{b}}$ \\
\hline UA $(\mu \mathrm{M})$ & I37.5 (103.0, 178.9) & $143.0(106.5,195.6)^{\mathrm{a}}$ & | 40.7 (I06.7, I82.9) & I $43.8(\mid 05.4,207.0)^{d}$ \\
\hline
\end{tabular}

Notes: All values are presented as the mean \pm SD or $\mathrm{n}(\%)$ or as the median (interquartile range); ${ }^{\mathrm{a}} \mathrm{P}<0.05$ for sepsis vs control; ${ }^{\mathrm{b}} \mathrm{P}<0.05$ for severe sepsis vs mild sepsis; ${ }^{\mathrm{C}} \mathrm{P}<0.05$ for mild sepsis vs control; ${ }^{\mathrm{d}} \mathrm{P}<0.05$ for severe sepsis vs control.

Abbreviations: SBP, systolic blood pressure; DBP, diastolic blood pressure; PCT, procalcitonin; CRP, C-reactive protein; AST, aspartate aminotransferase; ALT, alanine aminotransferase; BUN, blood urea nitrogen; CREA, creatinine; UA, uric acid; CPR, C-reactive protein-to-platelet ratio.

\section{Results}

\section{Patient Characteristics}

A total of 1098 neonates with suspected sepsis who met the inclusion criteria were enrolled in this study. Of the 1098 neonates, 599 were clinically diagnosed with sepsis; of which, 256 and 343 were diagnosed with mild sepsis and severe sepsis, respectively. The remaining 449 neonates without sepsis were classified as the infection group. Clinical characteristics and laboratory data of patients are provided in Table 1. Compared with neonates in the infection group, neonates with sepsis were older; had higher body temperature, respiratory rate and heart rate; and had higher levels of PCT, CRP, neutrophils, ALT, BUN, UA and CPR $(\mathrm{P}<0.001)$. However, the levels of PLT and CREA were significantly decreased $(\mathrm{P}<0.05)$ in neonates with sepsis. Based on the severity of sepsis, the neonates were divided into the following two groups: mild sepsis group and severe sepsis group. Further analysis showed that only PCT levels, CRP levels and CPR showed a significant gradual increase in the infection, mild sepsis and severe sepsis groups $(\mathrm{P}<0.05)$.

\section{Association of CPR with Neonatal Sepsis}

According to the CPR tertiles, we classified the neonates into three groups. As shown in Table 2, neonates in tertile 3 had higher level of PCT levels and neutrophils counts. 
Table 2 The Presence and Severity of Neonatal Sepsis Based on CPR Tertiles

\begin{tabular}{|l|c|c|c|}
\hline Variables & Tertile I (< 2.33) (n= 350) & Tertile 2 (2.33-5.52) (n= 349) & Tertile 3 (> 5.52) (n= 349) \\
\hline Age (days) & $11.0(7.0,16.0)$ & $6.0(4.0,13.0)^{\mathrm{a}}$ & $9.0(5.0,16.0)^{\mathrm{bc}}$ \\
\hline Male, $\mathrm{n}(\%)$ & $187(53.4 \%)$ & $226(64.8 \%)^{\mathrm{a}}$ & $224(64.2 \%)^{\mathrm{b}}$ \\
\hline PCT $(\mathrm{ng} / \mathrm{mL})$ & $0.12(0.09,0.18)$ & $0.17(0.11,0.35)^{\mathrm{a}}$ & $0.50(0.19,2.92)^{\mathrm{bc}}$ \\
\hline WBC $\left(10^{9}\right.$ cells/L) & $10.7(8.9,13.5)$ & $8.9(7.0,11.6)^{\mathrm{a}}$ & $10.1(6.8,14.2)^{\mathrm{bc}}$ \\
\hline Neutrophils $\left(10^{9}\right.$ cells/L) & $4.3(3.3,6.3)$ & $4.3(2.8,6.3)$ & $5.0(3.1,9.1)^{\mathrm{bc}}$ \\
\hline Clinical data & & & $64(18.3 \%)^{\mathrm{bc}}$ \\
\hline Infection, $\mathrm{n}(\%)$ & $194(55.4 \%)$ & $191(54,7 \%)$ & $285(81.7 \%)^{\mathrm{bc}}$ \\
\hline Overall sepsis, $\mathrm{n}(\%)$ & $156(44.6 \%)$ & $158(45.3 \%)$ & $96(27.5 \%)$ \\
\hline Mild sepsis, $\mathrm{n}(\%)$ & $81(23.1 \%)$ & $79(22.6 \%)$ & $189(54.2 \%)^{\mathrm{bc}}$ \\
\hline Severe sepsis, $\mathrm{n}(\%)$ & $75(21.4 \%)$ & $79(22.6 \%)$ & \\
\hline
\end{tabular}

Notes: ${ }^{\text {ap }}<0.05$ for Tertile 2 vs Tertile I; ${ }^{\text {P }}<<0.05$ for Tertile 3 vs Tertile I; ${ }^{\mathrm{P}}<0.05$ for Tertile 3 vs Tertile 2 .

Abbreviations: abbreviations as in Table I.

The prevalence of sepsis increased significantly from $44.6 \%$ in tertile 1 to $81.7 \%$ in tertile $3(\mathrm{P}<0.001)$, whereas the infection group was more likely to be in CPR tertile 1 and tertile 2. Further analysis revealed that the prevalence of severe sepsis was significantly higher in CPR tertile 3 than that in tertile 1 and tertile $2(\mathrm{P}<0.05)$.

\section{Predictive Value of CPR for Neonatal}

\section{Sepsis}

Univariate and multivariable binary logistic regression analyses were performed to evaluate the role of CPR in the diagnosis of neonatal sepsis (Table 3). After adjusting age; temperature; heart rate; respiratory rate; weight; neutrophil counts and the levels of PCT, ALB, ALP, AST, ALT, UREA and UA, CPR was proved to be an independent predictor of neonatal sepsis (odds ratio [OR], 1.015, 95\% confidence interval [CI], 1.008-1.022, $\mathrm{P}<0.001)$. Furthermore, our data also revealed that the tertiles of CPR were independently associated with an increased prevalence of neonatal sepsis. In addition, our data also confirmed that $\mathrm{CPR}$ and $\mathrm{CPR}$ tertiles were independent predictor of severe sepsis.

\section{Diagnostic Performance of the CPR}

ROC curve analysis was performed to evaluate the predictive value of CPR for sepsis. As shown in Figure 1A, the area under the ROC curves (AUC) showed a well discriminatory power of $\mathrm{CPR}$ (AUC $=0.68,95 \% \mathrm{CI}$, $0.65-0.72, \mathrm{P}<0.001)$ in predicting neonatal sepsis. The optimal diagnostic cut-off point was $5.41 \mathrm{mg} / 10^{6}$ cells, with $49 \%$ sensitivity and $86 \%$ specificity. In addition, we also analyzed the role of CPR in predicting severe sepsis. The AUC value for CPR in predicting severe sepsis was 0.68 (95\% CI, 0.64-0.72, $\mathrm{P}<0.001$ ) (Figure 1B). The optimal diagnostic cut-off point was $6.10 \mathrm{mg} / 10^{6}$ cells (with a sensitivity of $55 \%$ and specificity of $78 \%$ ).

\section{Discussion}

Neonatal sepsis is a serious life-threatening disease. Although the current diagnosis and treatment technologies have made significant progress, the diagnosis of neonatal sepsis still faces many challenges. For example, blood culture, the gold standard for diagnosis of neonatal sepsis, has a long laboratory turnaround time, which may cause a delay in diagnosis and the effective treatment. ${ }^{20}$ In addition, owing to the use of antibiotics before hospitalisation and an inadequate volume of blood, blood culture yields a low positive rate. ${ }^{20}$ Moreover, the clinical signs of neonatal sepsis are multiple and non-specific. ${ }^{6}$ Therefore, circulating blood biomarkers that may be useful in the early diagnosis of neonatal sepsis are under investigation. ${ }^{21}$

Sepsis is characterized by SIRS caused by pathogens infection, and biomarkers of inflammation play an important role in the diagnosis of neonatal sepsis. ${ }^{22,23}$ CRP is a well-known and commonly used marker, which was closely associated with inflammationrelated diseases. ${ }^{7}$ Various studies have demonstrated 
Table 3 Regression Analysis to Assess the Presence of Neonatal Sepsis and Severe Sepsis Based on CPR Tertiles

\begin{tabular}{|c|c|c|c|c|}
\hline \multirow[t]{2}{*}{ Variables } & \multicolumn{2}{|l|}{ Univariate } & \multicolumn{2}{|l|}{ Multivariate* } \\
\hline & OR $(95 \% \mathrm{CI})$ & $\mathbf{P}$ & OR $(95 \% \mathrm{CI})$ & $\mathbf{P}$ \\
\hline \multicolumn{5}{|c|}{ Presence of sepsis } \\
\hline CPR & $1.024(1.017-1.032)$ & $<0.001$ & $1.015(1.008-1.022)$ & $<0.001$ \\
\hline \multicolumn{5}{|c|}{ CPR tertiles } \\
\hline Tertile I & I & & 1 & \\
\hline Tertile 2 & $1.029(0.764-1.386)$ & 0.852 & $0.908(0.628-1.313)$ & 0.628 \\
\hline Tertile 3 & $5.538(3.928-7.807)$ & $<0.001$ & 2.331 (I.527-3.559) & $<0.001$ \\
\hline \multicolumn{5}{|c|}{ Presence of severe sepsis } \\
\hline CPR & $1.004(1.003-1.006)$ & $<0.001$ & $1.002(1.000-1.003)$ & 0.007 \\
\hline \multicolumn{5}{|c|}{ CPR tertiles } \\
\hline Tertile I & I & & 1 & \\
\hline Tertile 2 & $1.073(0.750-1.534)$ & 0.700 & $0.929(0.623-1.385)$ & 0.717 \\
\hline Tertile 3 & $4.331(3.111-6.030)$ & $<0.001$ & $1.918(1.291-2.850)$ & 0.001 \\
\hline
\end{tabular}

Notes: *Adjusted for age, temperature, heart rate, respiratory rate, weight, PCT, Neutrophil, ALB, ALP, AST, ALT, UREA and UA. Abbreviations: abbreviations as in Table I.

the CRP is an important prognostic factor for neonatal sepsis. $^{24-27}$ However, CRP exhibited a low specificity owing to its physiological increase after birth or the presence of non-infection-related conditions. ${ }^{11,28}$ PLTs are anucleate cells that play an important role in modulating haemostasis and developing thrombosis. ${ }^{29,30}$ Increasing evidences has revealed that PLTs played a critical role in inflammation and immune responses. ${ }^{31,32}$ PLTs can interacts with other leukocytes

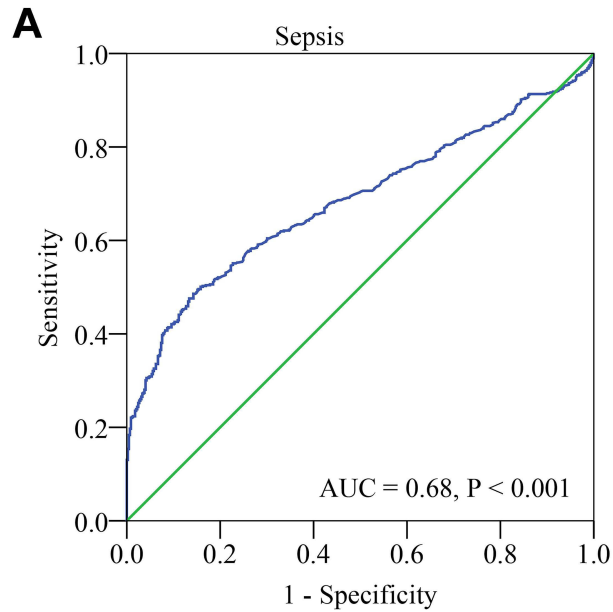

by expressing and secreting adhesion molecules and immune modulators, which further enhances inflammation. ${ }^{33-36}$ In addition to the hyperinflammation caused by PLTs, they can also catalyse the development of disseminated intravascular coagulation and microthrombosis, leading to sepsis complications and organ dysfunction. ${ }^{13}$ Clinical studies have demonstrated that a low PLT count is a well-known biomarker for disease severity of sepsis ${ }^{33,37}$ and an independent

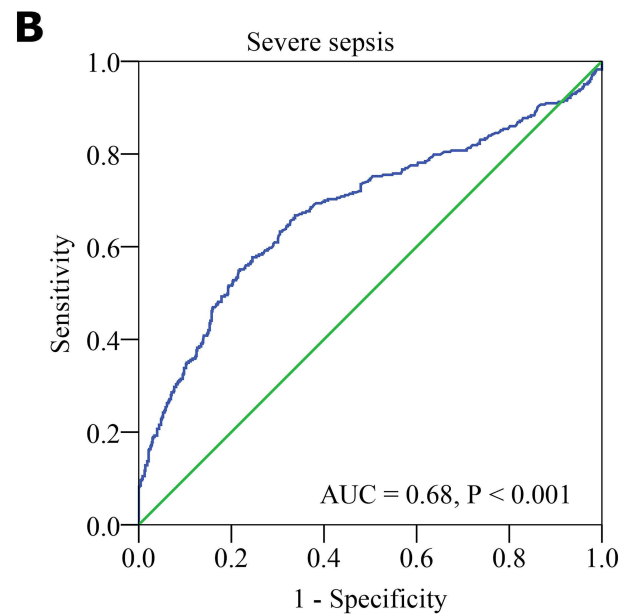

Figure I ROC curve of CPR in predicting sepsis and severe sepsis in neonates. (A) The ROC curve for CPR in predicting sepsis. (B) The ROC curve for CPR in predicting severe sepsis. 
prognostic predictor of 1-year overall survival of patients with sepsis. ${ }^{38}$

$\mathrm{CPR}$, as an emerging risk factor, can reflect both inflammation and coagulation status. However, there are no published studies regarding the relationship between CPR and neonatal sepsis. In this study, for the first time, we investigated the predictive role of CPR in neonatal sepsis based on a relatively large sample size. Our data revealed that the level of CPR was higher in neonates with sepsis and increased with the disease severity of sepsis. The neonates were divided into three groups based on the CPR tertiles. Further analysis revealed that the prevalence of sepsis was significantly higher in CPR tertile 3 (up to $81.7 \%$ ), than in CPR tertile 1 and tertile 2. Multivariate analysis revealed that the CPR was an independent predictor of the presence and severity of neonatal sepsis. The ROC curve analysis revealed that the CPR had a well discriminatory power in predicting sepsis and severe sepsis.

Limitations to the study include the fact that this is a retrospective single-center study, which may lead to some information and selection biases. Second, the diagnosis of neonatal sepsis was based on clinical features and was not confirmed by positive blood culture. Therefore, the incidence rate of sepsis may be underestimated or overestimated. Third, CPR was only measured at admission; serial CPR evaluation may be useful to further explore the dynamic correlation between CPR and neonatal sepsis. Lastly, all of the enrolled patients were neonates with suspected sepsis. Therefore, the findings of this study may not be applicable to other populations.

\section{Conclusions}

In conclusion, our study demonstrated that CPR was higher in neonates with sepsis and increased with the disease severity. After adjusting for other variables, CPR was independently associated with the presence and severity of neonatal sepsis. Our findings highlight the potential clinical value of CPR in predicting the risk of neonatal sepsis.

\section{Ethics Approval and Consent to Participate}

The study was conducted according to the guidelines of the Declaration of Helsinki and was approved by the Hospital Ethics Review Board of the Henan Children's Hospital. We confirmed that all the data were anonymized and maintained with confidentiality; therefore, the requirement for informed consent was waived because of the retrospective nature of the present study.

\section{Funding}

This study was funded by the Key Research, Development, and Promotion Projects of Henan Province (202102310132), and the Medical Science and Technology Project of Henan Province (LHGJ20190970, LHGJ20200642 and LHGJ20 200651).

\section{Disclosure}

The authors report no conflicts of interest in this work.

\section{References}

1. Liu L, Oza S, Hogan D, et al. Global, regional, and national causes of child mortality in 2000-13, with projections to inform post-2015 priorities: an updated systematic analysis. Lancet. 2015;385 (9966):430-440. doi:10.1016/s0140-6736(14)61698-6

2. Lozano R, Naghavi M, Foreman K, et al. Global and regional mortality from 235 causes of death for 20 age groups in 1990 and 2010: a systematic analysis for the Global Burden of Disease Study 2010. Lancet. 2012;380(9859):2095-2128. doi:10.1016/S0140-6736(12) 61728-0

3. Wang H, Liddell CA, Coates MM, et al. Global, regional, and national levels of neonatal, infant, and under-5 mortality during 1990-2013: a systematic analysis for the Global Burden of Disease Study 2013. Lancet. 2014;384(9947):957-979. doi:10.1016/S01406736(14)60497-9

4. Scheer CS, Fuchs C, Gründling M, et al. Impact of antibiotic administration on blood culture positivity at the beginning of sepsis: a prospective clinical cohort study. Clin Microbiol Infect. 2019;25 (3):326-331. doi:10.1016/j.cmi.2018.05.016

5. Lamy B, Dargère S, Arendrup MC, Parienti JJ, Tattevin P. How to optimize the use of blood cultures for the diagnosis of bloodstream infections? A state-of-the art. Front Microbiol. 2016;7:697. doi:10.3389/fmicb.2016.00697

6. Shane AL, Sánchez PJ, Stoll BJ. Neonatal sepsis. Lancet. 2017;390 (10104):1770-1780. doi:10.1016/s0140-6736(17)31002-4

7. Sproston NR, Ashworth JJ. Role of C-reactive protein at sites of inflammation and infection. Front Immunol. 2018;9:754. doi:10.3389/fimmu.2018.00754

8. Povoa P, Almeida E, Moreira P, et al. C-reactive protein as an indicator of sepsis. Intensive Care Med. 1998;24(10):1052-1056. doi:10.1007/s001340050715

9. Stocker M, van Herk W, El Helou S, et al. C-reactive protein, procalcitonin, and white blood count to rule out neonatal early-onset sepsis within 36 hours: a secondary analysis of the neonatal procalcitonin intervention study. Clin Infect Dis. 2020. doi:10.1093/cid/ciaa876

10. Wang HE, Shapiro NI, Safford MM, et al. High-sensitivity C-reactive protein and risk of sepsis. PLoS One. 2013;8(7):e69232. doi:10.1371/ journal.pone.0069232

11. Hofer N, Zacharias E, Muller W, Resch B. An update on the use of C-reactive protein in early-onset neonatal sepsis: current insights and new tasks. Neonatology. 2012;102(1):25-36. doi:10.1159/000336629

12. Khan F. C-reactive protein as a screening biomarker in neonatal sepsis. J Coll Physicians Surg Pak. 2019;29(10):951-953. doi: $10.29271 /$ jepsp.2019.10.951 
13. de Stoppelaar SF, van't Veer C, van der Poll T. The role of platelets in sepsis. Thromb Haemost. 2014;112(4):666-677. doi:10.1160/TH14-020126

14. Greco E, Lupia E, Bosco O, Vizio B, Montrucchio G. Platelets and multi-organ failure in sepsis. Int J Mol Sci. 2017;18(10):2200. doi:10.3390/ijms18102200

15. Russwurm S, Vickers J, Meier-Hellmann A, et al. Platelet and leukocyte activation correlate with the severity of septic organ dysfunction. Shock. 2002;17(4):263-268. doi:10.1097/00024382200204000-00004

16. Guclu E, Durmaz Y, Karabay O. Effect of severe sepsis on platelet count and their indices. Afr Health Sci. 2013;13(2):333-338. doi:10.4314/ahs.v13i2.19

17. Claushuis TA, van Vught LA, Scicluna BP, et al. Thrombocytopenia is associated with a dysregulated host response in critically ill sepsis patients. Blood. 2016;127(24):3062-3072. doi:10.1182/blood-201511-680744

18. Goldstein B, Giroir B, Randolph A. International pediatric sepsis consensus conference: definitions for sepsis and organ dysfunction in pediatrics. Pediatr Crit Care Med. 2005;6(1):2-8. doi:10.1097/01. Pcc.0000149131.72248.E6

19. Li T, Dong G, Zhang M, et al. Association of neutrophil-lymphocyte ratio and the presence of neonatal sepsis. $J$ Immunol Res. 2020;2020:7650713. doi:10.1155/2020/7650713

20. Iroh Tam PY, Bendel CM. Diagnostics for neonatal sepsis: current approaches and future directions. Pediatr Res. 2017;82(4):574-583. doi:10.1038/pr.2017.134

21. Sharma D, Farahbakhsh N, Shastri S, Sharma P. Biomarkers for diagnosis of neonatal sepsis: a literature review. J Matern Fetal Neonatal Med. 2018;31(12):1646-1659. doi:10.1080/14767058.2017.1322060

22. Opal SM, Wittebole X. Biomarkers of Infection and Sepsis. Crit Care Clin. 2020;36(1):11-22. doi:10.1016/j.ccc.2019.08.002

23. Li T, Zhang Z, Li X, et al. Neutrophil extracellular traps: signaling properties and disease relevance. Mediators Inflamm. 2020;2020:9254087. doi:10.1155/2020/9254087

24. Ruan L, Chen GY, Liu Z, et al. The combination of procalcitonin and C-reactive protein or presepsin alone improves the accuracy of diagnosis of neonatal sepsis: a meta-analysis and systematic review. Crit Care. 2018;22(1):316. doi:10.1186/s13054-018-2236-1

25. Omran A, Maaroof A, Saleh MH, Abdelwahab A. Salivary C-reactive protein, mean platelet volume and neutrophil lymphocyte ratio as diagnostic markers for neonatal sepsis. J Pediatr. 2018;94 (1):82-87. doi:10.1016/j.jped.2017.03.006
26. Liu Y, Zhao L, Wu Z. Accuracy of C-reactive protein test for neonatal septicemia: a diagnostic meta-analysis. Med Sci Monit. 2019;25:4076-4081. doi:10.12659/MSM.916968

27. Li T, Li X, Wei Y, et al. Predictive value of C-reactive protein-toalbumin ratio for neonatal sepsis. $J$ Inflamm Res. 2021;14:3207-3215. doi:10.2147/JIR.S321074

28. Eschborn S, Weitkamp JH. Procalcitonin versus C-reactive protein: review of kinetics and performance for diagnosis of neonatal sepsis. J Perinatol. 2019;39(7):893-903. doi:10.1038/s41372-019-0363-4

29. Gremmel T, Frelinger AL, Michelson AD. Platelet physiology. Semin Thromb Hemost. 2016;42(3):191-204. doi:10.1055/s-0035-1564835

30. Hvas AM. Platelet function in thrombosis and hemostasis. Semin Thromb Hemost. 2016;42(3):183-184. doi:10.1055/s-0036-1572329

31. Koupenova M, Clancy L, Corkrey HA, Freedman JE. Circulating platelets as mediators of immunity, inflammation, and thrombosis. Circ Res. 2018;122(2):337-351. doi:10.1161/CIRCRESAHA.117.310795

32. Thomas MR, Storey RF. The role of platelets in inflammation. Thromb Haemost. 2015;114(3):449-458. doi:10.1160/TH14-12-1067

33. Morrell CN, Aggrey AA, Chapman LM, Modjeski KL. Emerging roles for platelets as immune and inflammatory cells. Blood. 2014;123(18):2759-2767. doi:10.1182/blood-2013-11-462432

34. Elzey BD, Tian J, Jensen RJ, et al. Platelet-mediated modulation of adaptive immunity. A communication link between innate and adaptive immune compartments. Immunity. 2003;19(1):9-19. doi:10.1016/ s1074-7613(03)00177-8

35. Semple JW, Italiano JE, Freedman J. Platelets and the immune continuum. Nat Rev Immunol. 2011;11(4):264-274. doi:10.1038/nri2956

36. Sonmez O, Sonmez M. Role of platelets in immune system and inflammation. Porto Biomed J. 2017;2(6):311-314. doi:10.1016/j. pbj.2017.05.005

37. Thiery-Antier N, Binquet C, Vinault S, et al. Is thrombocytopenia an early prognostic marker in septic shock? Crit Care Med. 2016;44 (4):764-772. doi:10.1097/CCM.0000000000001520

38. Zhao L, Zhao L, Wang YY, et al. Platelets as a prognostic marker for sepsis: a cohort study from the MIMIC-III database. Medicine. 2020;99(45):e23151. doi:10.1097/MD.0000000000023151
Journal of Inflammation Research

\section{Publish your work in this journal}

The Journal of Inflammation Research is an international, peerreviewed open-access journal that welcomes laboratory and clinica findings on the molecular basis, cell biology and pharmacology of inflammation including original research, reviews, symposium reports, hypothesis formation and commentaries on: acute/chronic inflammation; mediators of inflammation; cellular processes; molecular mechanisms; pharmacology and novel anti-inflammatory drugs; clinical conditions involving inflammation. The manuscript management system is completely online and includes a very quick and fair peerreview system. Visit http://www.dovepress.com/testimonials.php to read real quotes from published authors. 